\title{
Assessment of Spatial Heterogeneity of Ventricular Repolarization After Quinidine in Healthy Subjects
}

\author{
Valentina D.A. Corino ${ }^{1}$, Massimo W. Rivolta ${ }^{2}$, Luca T. Mainardi ${ }^{1}$, Roberto Sassi ${ }^{2}$ \\ ${ }^{1}$ Dipartimento di Elettronica, Informazione e Bioingegneria, Politecnico di Milano, Milan, Italy \\ ${ }^{2}$ Dipartimento di Informatica, Universitá degli Studi di Milano, Italy
}

\begin{abstract}
When spatial heterogeneity of ventricular repolarization (SHVR) increases, vulnerability to ventricular arrhythmias, including lethal ones, has also been observed to increase. Drug-induced multi-ion-channel blocks may increase SHVR. Aim of this study is to non-invasively assess whether quinidine, a strong hERG potassium channel blocker with weaker effects on calcium and late sodium currents, increases SHVR. We analyzed data from 21 healthy subjects that received both the drug and a placebo and underwent to 12 leads Holter monitoring. From the recording, three 10-s ECGs were extracted at each of 16 predefined time-points. SHVR was assessed by the $\mathcal{V}-$ index, which evaluates the standard deviation of the repolarization times from multi-lead ECG recordings. At any time point, a value of $\mathcal{V}$-index was computed for each of the three 10s ECGs and averaged if the difference in the mean RR of the 10s ECGs was lower than $50 \mathrm{~ms}$. The $\mathcal{V}-$ index did not change after the placebo $(\mathcal{V}$-index pre-dose $=29.2 \pm 9.9 \mathrm{~ms}$ vs. $\mathcal{V}$-index post-dose $1 \mathrm{~h}=26.7 \pm 10.3$, $n s)$, whereas, after quinidine, it significantly increased one hour post-dose $(\mathcal{V}$-index pre-dose $=29.5 \pm 10.2 \mathrm{~ms} v \mathrm{v} . \mathcal{V}-$ index post-dose $1 \mathrm{~h}=46.5 \pm 33.8 \mathrm{~ms}, \mathrm{p}=0.01)$. Quinidine had its maximum effect on the $\mathcal{V}$-index $2.5 \mathrm{~h}$ after dose $(\mathcal{V}$-index post-dose $2.5 \mathrm{~h}=53.6 \pm 39.6 \mathrm{~ms})$.
\end{abstract}

\section{Introduction}

When spatial heterogeneity of ventricular repolarization (SHVR) increases, vulnerability to ventricular arrhythmias, including lethal ones, has also been observed to increase [1]. At the cell level, it is well known that the ion channel abnormalities are associated with the genesis of lethal arrhytmias as torsade de pointes or ventricular fibrillation. It is important to identify drugs that block relevant ion channels. The most significant block occurs to the human ether-á-go-go-related gene (hERG) potassium channel (an outward current) and prolongs the QT interval on the electrocardiogram (ECG) [2,3]. However, a drug blocking the hERG potassium channel may not be associated to a high torsade risk because it may also block other channels (calcium and/or sodium, inward currents).

To estimate heterogeneity of ventricular repolarization or spatial dispersion of ventricular repolarization, the $\mathcal{V}-$ index has been recently introduced [4]: the $\mathcal{V}$-index is a measure that provides an estimate of the standard deviation of the repolarization times of the myocytes across the entire myocardium from the surface ECG. The $\mathcal{V}$-index has been previously used to assess ventricular repolarization during moxifloxacin and solatol [5], and also in patients with Chagas disease, showing an increased dispersion of repolarization times correlated with the risk of death in a univariate survival analysis [6].

Aim of this study is to non-invasively assess whether quinidine, a strong hERG potassium channel blocker with weaker effects on calcium and late sodium currents, increases SHVR, as estimated by the $\mathcal{V}$-index.

\section{Material and methods}

\subsection{Protocol}

The design of this clinical study has been previously described [7]. Briefly, 22 healthy young subjects (27 \pm 6 years, range 18-35, 11 males) without a family history of cardiovascular disease were included in the study. The subjects received a single dose of $400 \mathrm{mg}$ quinidine sulfate (Watson Pharma, Corona, CA) or placebo under fasting conditions. Quinidine, not only blocks the hERG potassium channel, but also calcium and sodium channels at high concentrations [8].

During each period, standard 12-lead continuous ECGs were recorded at $500 \mathrm{~Hz}$ and then upsampled at $1 \mathrm{kHz}$. From the continuous recording, three 10-second ECGs were extracted at predose and 15 predefined time points post-dose $(0.5,1,1.5,2,2.5,3,3.5,4,5,6,7,8,12$, 14 , and $24 \mathrm{~h}$ ) during which the subjects were resting in a supine position for 10 minutes and made available for further analysis. Data are available on physionet.org [9]. 


\section{2. $\mathcal{V}$-index}

Myocytes' transmembrane potential (TMP) morphology and, particularly, durations differ when traversing the heart from apex to base and across the muscular tissue from the endo- to the epicardium. However, in first approximation, the slope of the TMP during phase 3 does not differ significantly across myocytes. Let's divide the myocardium in $M$ nodes and let's suppose that each node $m$ shares the same TMP during repolarization, which we represent here with a common function $D\left(t-\rho_{m}(k)\right)$, where $\rho_{m}(k)$ marks the repolarization time of the $k^{t h}$ beat, as the point where the down-slope is maximal. $\rho_{m}(k)$ may be expressed as

$$
\rho_{m}(k)=\bar{\rho}(k)+\Delta \rho_{m}(k),
$$

where the repolarization delay $\Delta \rho_{m}(k)$ is the deviation from the average repolarization time $\bar{\rho}(k)=$ $\frac{1}{M} \sum_{m=1}^{M} \rho_{m}(k)$ in the given heartbeat $k$.

Sassi and Mainardi [4] introduced a simple model to describe the distribution of these delays:

$$
\Delta \rho_{m}(k)=\vartheta_{m}+\varphi_{m}(k),
$$

where $\vartheta_{m}$ models the spatial variability of the repolarization times for a given subject at a given heart rate, and $\varphi_{m}(k)$ describes temporal differences in repolarization times which are observable among successive beats.

Under a few (common) hypotheses, usually enforced in forward and inverse electrocardiographical solversthe link between $\Delta \rho_{m}(k)$ and the T-wave $\boldsymbol{\Psi}(t)$ on the ECG (being $\Psi(t)$ a $L \times 1$ vector containing the T-wave values for each lead) can be derived by analytically simplifying a biophysical model [10]. Specifically,

$$
\begin{aligned}
\mathbf{\Psi}(t) & \approx-\mathbf{A} \Delta \rho T_{d}(t)+12 \mathbf{A} \Delta \rho^{2} \dot{T}_{d}(t) \\
& =\mathbf{w}_{\mathbf{1}} \mathbf{T}_{\mathbf{d}}(\mathbf{t})+\mathbf{w}_{\mathbf{2}} \dot{\mathbf{T}}_{\mathbf{d}}(\mathbf{t}),
\end{aligned}
$$

where the function $T_{d}(t)$ is the first derivative of $D(t)$ (which, with a sign reversal, is often termed "dominant Twave" [10]) and $\Delta \rho=\left[\Delta \rho_{1}(k), \Delta \rho_{2}(k), \ldots, \Delta \rho_{M}(k)\right]^{T}$ is a vector of repolarization delays. $\mathbf{A}$ is a patientdependent $[L \times M]$ transfer matrix accounting for the contribution of each node to the $L$-leads electrocardiographic recording in $\boldsymbol{\Psi}(t)$. The terms $\mathbf{w}_{1}$ and $\mathbf{w}_{2}$ are $[L \times 1]$ vector of scalars ("lead factors"), one for each beat.

An estimate of the SHVR, quantified as the sample standard deviation of the repolarization times across the myocardium, can be derived from the the lead factors through the $\mathcal{V}$-index, defined as:

$$
\mathcal{V}_{i}=\frac{\operatorname{std}\left[\mathrm{w}_{2}(\mathrm{i})\right]}{\operatorname{std}\left[\mathrm{w}_{1}(\mathrm{i})\right]} \approx s_{\vartheta}=\left(\frac{1}{M} \sum_{m=1}^{M} \vartheta_{m}^{2}\right)^{1 / 2},
$$

where the standard deviations (std) are computed on the lead factors of lead $i$ across a certain number of consecutive beats (not across different leads).

SHVR, as measured by the $\mathcal{V}$-index, has a straightforward physiological interpretation and does not suffer from an imperfect location of ECG fiducial points [4]. Moreover it was proved to be consistent by extensive numerical simulations $[4,5]$ and promising preliminary clinical validations $[6,11]$.

\subsection{Preprocessing}

Standard ECG preprocessing was performed in four steps. First, ECG signals were bandpass filtered (3rd order, Butterworth, $0.540 \mathrm{~Hz}$, zero-phase forward and reverse filtering) to reduce baseline wandering and high frequency noise. Second, for each lead independently, the isoelectric line was approximately set to $0 \mathrm{mV}$ by subtracting a straight line estimated by linear regression of the points belonging to the TP segments (these points were identified through a binning of the ECGs amplitude distribution) [5]. Third, beats were detected by means of an ad-hoc implementation of the Pan-Tompkins detector on lead II and then, a QRS template was used to re-align the beats, using a cross-correlation-based algorithm, to obtain a common fiducial point. Fourth, lead quality was assessed by the average cross-correlation between the QRS template and the aligned QRS complexes. Leads with an average cross-correlation higher than 0.9 were considered of enough quality and then, further analyzed.

The $\mathcal{V}$-index was computed on ECG signals having at least three high quality leads (out of 12) by means of an iterative numerical algorithm previously validated [6]. Briefly, the algorithm estimated alternatively the lead factors (i.e., $\mathbf{w}_{\mathbf{1}}$ and $\mathbf{w}_{\mathbf{2}}$ ) and on each T-wave, using a discretized version of the Eulero-Lagrange equations. In this way, we computed one $\mathcal{V}$-index value for each high quality lead. We considered their average as an overall estimate of the $\mathcal{V}$-index. Since each ECG recording lasted $10 \mathrm{~s}$, the number of beats available for the $\mathcal{V}$-index computation was rather limited. To increase the robustness of the estimates in a specific time-point, we averaged the $\mathcal{V}$-index values obtained in the three ECG replica close to that time point. Given the fact that the $\mathcal{V}$-index might depends on the heart rate, the values were averaged only when the corresponding averaged RR did not differ more than $50 \mathrm{~ms}$ from each other (i.e., the heart rate was stable).

\subsection{Statistical analysis}

One-way repeated-measures Friedman test was performed to compare the parameters over time; if the p-value of the Friedman test was significant, a paired Wilcoxon test with Holms correction was applied. Paired t-test or 
(a)

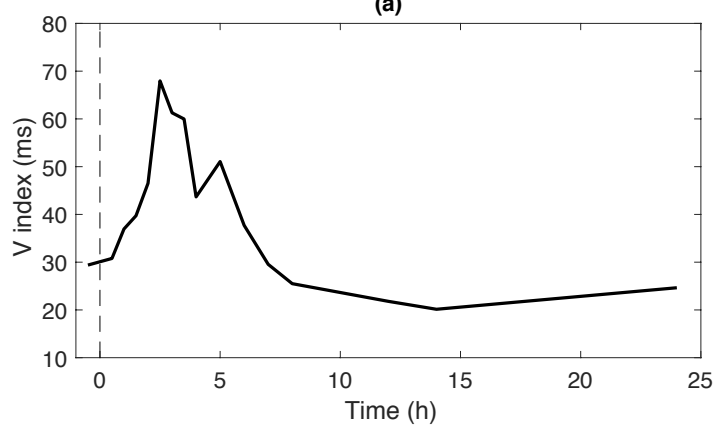

(b)

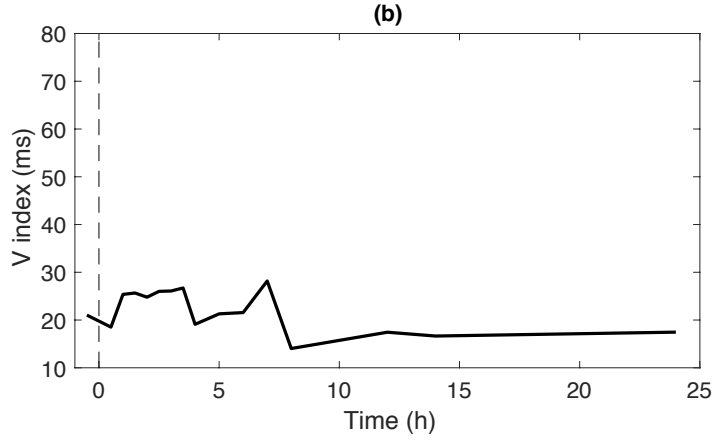

Figure 1. Example of $\mathcal{V}$-index for one subject over time during (a) quinidine and (b) placebo administration. The dash vertical line indicates the time of quinidine or placebo administration.

Wilcoxon test was also used to compare drugs and placebo parameters at the same time point. $\mathrm{P}$ values of $<0.05$ were considered statistically significant. All analyses and statistical tests were performed using MATLAB R2016a (The MathWorks).

\section{Results}

The heart rate in each time point is quite stable for the three repetitions, with a range of the RR mean of $32 \pm 18$ $\mathrm{ms}$ for quinidine and $31 \pm 18 \mathrm{~ms}$ for the placebo. Only $6 \%$ and $4 \%$ of the segments were excluded because of the lack of at least two repetitions with similar RR, for quinidine and the placebo, respectively.

Figure 1 shows the $\mathcal{V}$-index for one subject during (a) quinidine and (b) placebo administration. It can be observed that the placebo does not affect the $\mathcal{V}$-index. On the contrary, after the administration of the quinidine, an increase of $\mathcal{V}$-index is observed, going from 29 to $68 \mathrm{~ms}$, from pre-dose time point to the peak. The peak of $\mathcal{V}$-index after quinidine administration is observed after 2.5 hours.

This result is confirmed on the whole population, as shown in Figure 2. It can be observed that quinidine leads to a significant $\mathcal{V}$-index increase, whereas there is no effect
Table 1. Percentage of increase at the peak with respect to pre-dose values.

\begin{tabular}{lc}
\hline \hline Parameter & \% increase \\
\hline & \\
$\mathcal{V}$-index & $143 \pm 111$ \\
QTc & $26 \pm 6$ \\
Tpeak-Tend & $83 \pm 45$ \\
J-Tpeak & $7 \pm 6$ \\
\hline \hline
\end{tabular}

of the placebo. In particular, during quinidine, comparing the value of $\mathcal{V}$-index at each time point with the pre-dose one, a significant $(\mathrm{p}<0.05)$ increase can be observed after one hour of quinidine. Moreover, in Figure 2 the average concentration of the drug is shown: it can be observed that the peak of $\mathcal{V}$-index is at the same time point as the peak of the drug concentration appears. No difference in the $\mathcal{V}$-index is observed after the placebo administration.

Finally, Table 1 shows the percentage of increase of $\mathcal{V}-$ index (measured at the peak) and three classical parameters (obtained together with the dataset) to assess ventricular repolarization heterogeneity, namely, the corrected QT interval (QTc), the interval between the peak and the end of $\mathrm{T}$ wave (Tpeak-Tend) and the interval between the Jpoint to the peak of T-wave (J-Tpeak). It can be observed that the increase of the $\mathcal{V}$-index is much larger than that of QTc, Tpeak-Tend, J-Tpeak.

\section{Conclusions}

These preliminary results show that the $\mathcal{V}$-index can recognize changes in SHVR due to quinidine. The percentage of increase of $\mathcal{V}$-index (measured at the peak) is much larger than that of three classical parametersto assess ventricular repolarization heterogeneity. The short duration of the analyzed recordings (a limitation of this study) may be the cause of the high standard deviation observed in the results, however, these may be reduced when analyzing longer recordings.

\section{References}

[1] Napolitano C, Priori SG, Schwartz PJ. Torsade de pointes. Mechanisms and management. Drugs 1994;47: 51-65.

[2] Stockbridge N, Morganroth J, Shah RR, Garnett C. Dealing with global safety issues: was the response to QTliability of non-cardiac drugs well coordinated? Drug Saf 2013;36:167-182.

[3] Trudeau MC, Warmke JW, Ganetzky B, Robertson GA. HERG, a human inward rectifier in the voltage-gated potassium channel family. Science 1995;269:92-95.

[4] Sassi R, Mainardi LT. An estimate of the dispersion of repolarization times based on a biophysical model of the ECG. IEEE Trans Biomed Eng. 2011;58:3396-3405. 
(a)

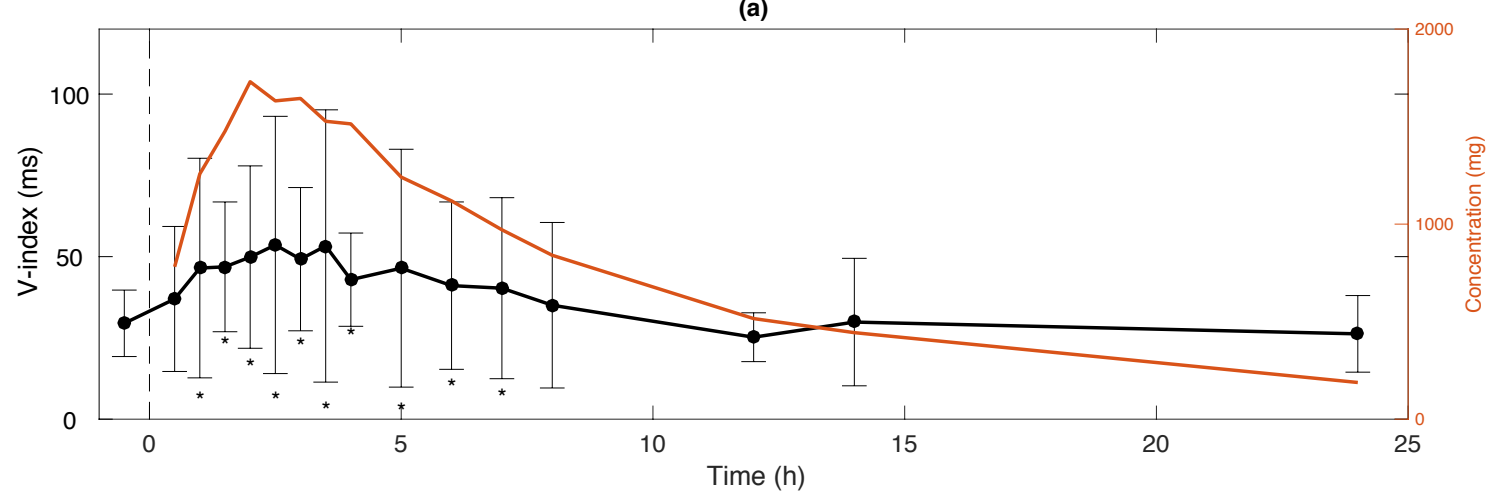

(b)

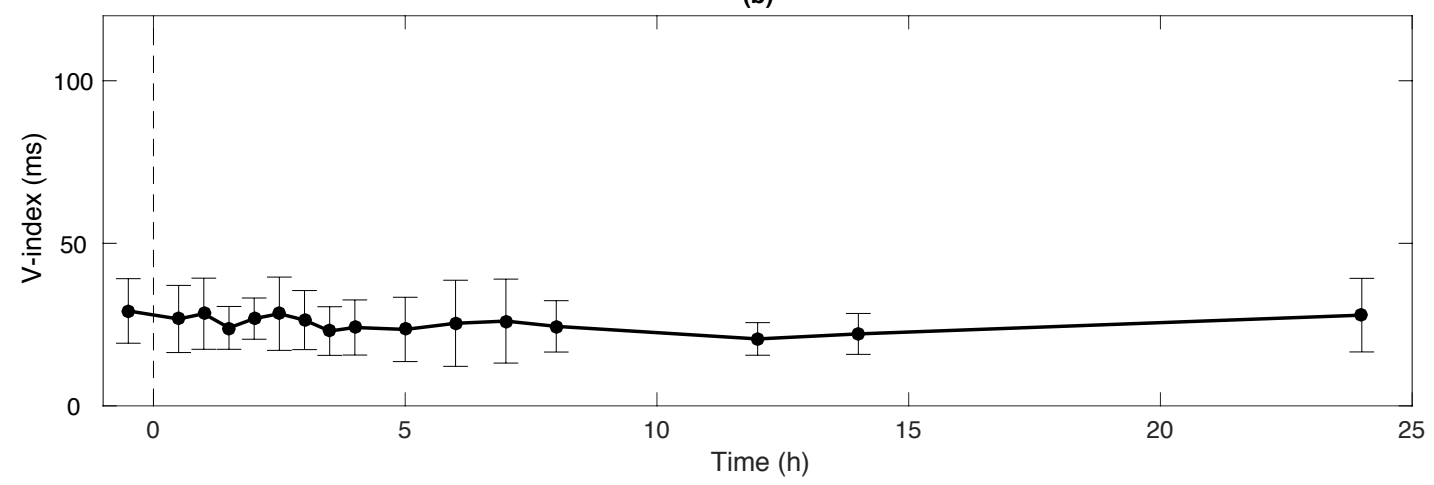

Figure 2. Average of $\mathcal{V}$-index for all the subjects over time during (a) quinidine and (b) placebo administration. The dash vertical line indicates the time of quinidine or placebo administration. $* \mathrm{p}<0.05$ when comparing the $\mathcal{V}$-index of a time point versus the pre-dose value. The average plasma concentration is superimposed (red line) in (a).

[5] Rivolta MW, Mainardi LT, Sassi R. Quantification of ventricular repolarization heterogeneity during moxioxacin or sotalol administration using V-index. Physiol Meas. 2015;36:803-811.

[6] Sassi R, Rivolta MW, Mainardi LT, Reis RC, Rocha MO, Ribeiro AL, Lombardi F. Spatial repolarization heterogeneity and survival in Chagas disease.Methods Inf Med. 2014;53:464-468.

[7] Johannesen L, Vicente J, Mason JW, Sanabria C, WaiteLabott K, Hong M, Guo P, Lin J, Sorensen JS, Galeotti L, Florian J, Ugander M, Stockbridge N, Strauss DG. Differentiating drug-induced multichannel block on the electrocardiogram: randomized study of dofetilide, quinidine, ranolazine, and verapamil. Clin Pharmacol Ther 2014;96:549-558.

[8] Kramer J, Obejero-Paz CA, Myatt G, Kuryshev YA, Bruening-Wright A, Verducci JS, Brown AM. MICE models: superior to the HERG model in predicting Torsade de Pointes. Sci Rep 2013;3: 2100.

[9] Goldberger AL, Amaral LAN, Glass L, Hausdorff JM, Ivanov PCh, Mark RG, Mietus JE, Moody GB, Peng CK, Stanley HE. PhysioBank, PhysioToolkit, and PhysioNet:
Components of a New Research Resource for Complex Physiologic Signals. Circulation 2000;101:e215-e220.

[10] van Oosterom A. Genesis of the $T$ wave as based on an equivalent surface source model. J Electrocardiol. 2001;34 Suppl:217-27.

[11] Abacherli R, Twerenbold R, Boeddinghaus J, Nestelberger T, MŁchler P, Sassi R, Rivolta MW, Roonizi EK, Mainardi LT, Kozhuharov N, Rubini Gimnez M, Wildi K, Grimm K, Sabti Z, Hillinger P, Puelacher C, Strebel I, Cupa J, Badertscher P, Roux I, Schmid R, Leber R, Osswald S, Mueller C, Reichlin T. Diagnostic and prognostic values of the V-index, a novel ECG marker quantifying spatial heterogeneity of ventricular repolarization, in patients with symptoms suggestive of non-ST-elevation myocardial infarction. Int J Cardiol 2017;236:23-29.

Address for correspondence:

Valentina D.A. Corino via Golgi 39, 20133 Milan (Italy)

valentina.corino@polimi.it 